ARTICLE

DOI: $10.1038 / s 41467-017-02237-1$

\title{
Evidence for topological type-II Weyl semimetal $\mathrm{WTe}_{2}$
}

\author{
Peng $\mathrm{Li}^{1}$, Yan Wen ${ }^{1}$, Xin He${ }^{1}$, Qiang Zhang ${ }^{1}$, Chuan Xia (i) ${ }^{1}$, Zhi-Ming Yu (i) ${ }^{2}$, Shengyuan A. Yang (1) ${ }^{2}$, \\ Zhiyong Zhu ${ }^{3}$, Husam N. Alshareef (1) ${ }^{1} \&$ Xi-Xiang Zhang (i) ${ }^{1}$
}

Recently, a type-II Weyl fermion was theoretically predicted to appear at the contact of electron and hole Fermi surface pockets. A distinguishing feature of the surfaces of type-II Weyl semimetals is the existence of topological surface states, so-called Fermi arcs. Although $\mathrm{WTe}_{2}$ was the first material suggested as a type-II Weyl semimetal, the direct observation of its tilting Weyl cone and Fermi arc has not yet been successful. Here, we show strong evidence that $\mathrm{WTe}_{2}$ is a type-II Weyl semimetal by observing two unique transport properties simultaneously in one $\mathrm{WTe}_{2}$ nanoribbon. The negative magnetoresistance induced by a chiral anomaly is quite anisotropic in $\mathrm{WTe}_{2}$ nanoribbons, which is present in $b$-axis ribbon, but is absent in $a$-axis ribbon. An extra-quantum oscillation, arising from a Weyl orbit formed by the Fermi arc and bulk Landau levels, displays a two dimensional feature and decays as the thickness increases in $\mathrm{WTe}_{2}$ nanoribbon.

\footnotetext{
${ }^{1}$ King Abdullah University of Science and Technology (KAUST), Physical Science and Engineering Division (PSE), Thuwal 23955-6900, Saudi Arabia. ${ }^{2}$ Research Laboratory for Quantum Materials, Singapore University of Technology and Design, Singapore 487372, Singapore. ${ }^{3}$ King Abdullah University of Science and Technology (KAUST), KAUST Supercomputing Laboratory (KSL), Thuwal 23955-6900, Saudi Arabia. Correspondence and requests for materials should be addressed to X.-X.Z. (email: xixiang.zhang@kaust.edu.sa)
} 
T he three-dimensional (3D) topological semimetals, Dirac and Weyl, have been extensively investigated as representatives of a new state of topological quantum matter that, in the bulk state, possesses electrons with linear dispersion at the Dirac or Weyl points ${ }^{1-17}$. Owing to the unique properties of these electrons in the bulk state (e.g., massless and defined chirality), other exotic physical properties have also been discovered, e.g., an unsaturated, positive magnetoresistance (MR) and an ultrahigh mobility of the carriers ${ }^{5,18}$. In addition to having unique bulk state electrons and novel topological surface states, the socalled Fermi arcs also exist on these surfaces with broken inversion symmetry ${ }^{6}$. A new quantum oscillation frequency originating from these Fermi arcs, observed in the Dirac semimetal $\mathrm{Cd}_{3} \mathrm{As}_{2}$, is also predicted to exist in ultrathin slabs of topological Dirac and Weyl semimetals ${ }^{12,19}$.

$\mathrm{WTe}_{2}$ was suggested as the first material candidate for a type-II Weyl semimetal, in which the Weyl points occur at the crossing of the oblique conduction and the valance bands (Weyl cone tilts along $Y$ axis in momentum space, Fig. 1a) ${ }^{6}$. It was also predicted that there exist eight separated Weyl points in the bulk of $\mathrm{WTe}_{2}$ and Fermi arcs on the (001) crystal surfaces owing to reflection symmetry. These Weyl points with opposite chiralities are possibly connected by Fermi arcs with a separation of $0.032 \mathrm{~A}^{-1}$ along $Y$ axis in momentum space (Fig. 1a) ${ }^{13,14}$. According to the definition of a type-II Weyl semimetal, a quasiparticle can be regarded as a type-II Weyl fermion that has the following characteristics. Along the $k$ direction that Weyl cone tilts toward, its kinetic energy is larger than its potential one in low-energy condensed matter physics ${ }^{6}$. More specially, type-II Weyl fermions violate the Lorentz symmetry strongly 6,20 . Recently, it was reported that the decisive criterion for a type-II Weyl semimetal is the direct observation of its tilted band crossing in three directions in the momentum space, as demonstrated in $\mathrm{LaAlGe}^{20}$. However, experimental demonstrations of type-II Weyl fermions in $\mathrm{WTe}_{2}$ have been unsuccessful owing to the limited resolution of angle-resolved photoemission spectroscopy (ARPES) and scanning tunneling spectroscopy, which prevents direct observation of the tilting Weyl cone and Fermi arcs in $\mathrm{WTe}_{2}{ }^{8,13,14,21}$.

It has been established that the anisotropy of chiral anomaly should be a strong evidence to distinguish the type-II Weyl semimetals from type-I Weyl semimetals, in addition of the direct observation of the tilted bulk Weyl cones using ARPES ${ }^{6,22}$. Chiral anomaly refers to the state at which the particle number with given chirality is non-conserved. A negative MR caused by this chiral anomaly should be observed when a magnetic field is applied parallel to the electric field ${ }^{23}$. In type-I Weyl semimetals, the chiral anomaly always appears regardless of the direction of the applied magnetic fields. However, in type-II Weyl semimetals, the chiral Landau levels and chiral anomaly exists only when the magnetic field is applied in the direction along which the kinetic energy of the fermions is larger than their potential energy $6,22,24-26$. Specifically, the ratio of the kinetic energy $\left(T_{\mathrm{k}}\right)$ to potential energy $\left(U_{\mathrm{k}}\right), R=T^{2}(\mathrm{k}) / U^{2}(\mathrm{k})>1$ was proposed to be a criterion to identify the directions along which a Weyl fermion is a

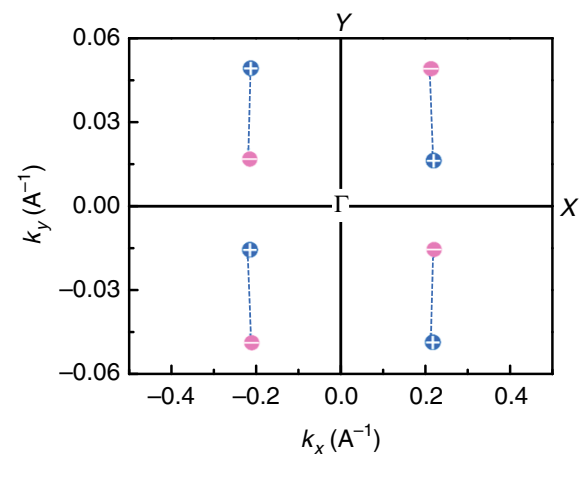

e

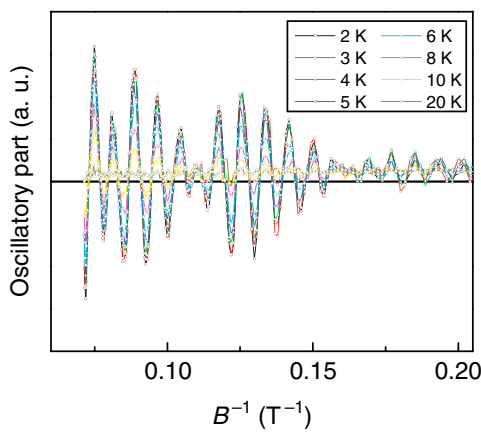

b

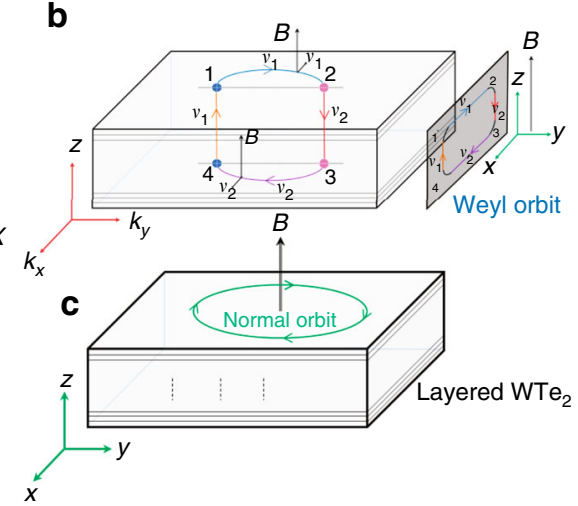

f

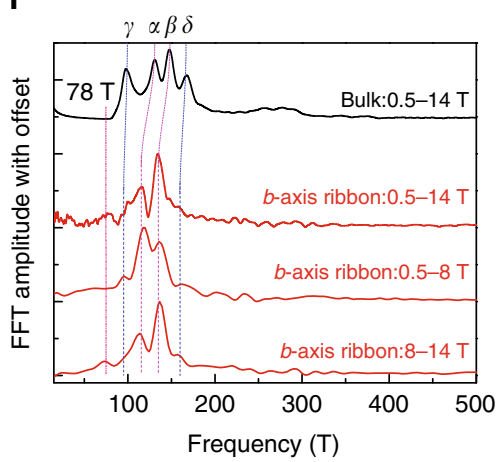

d

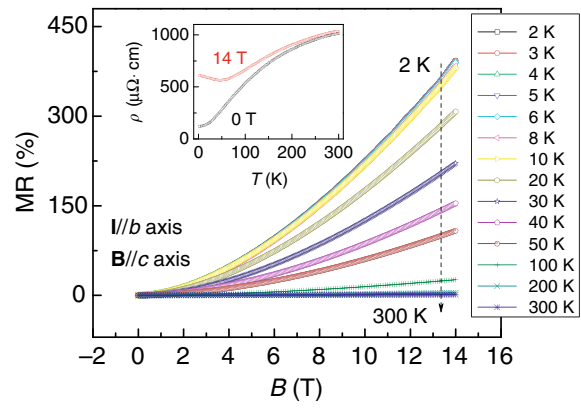

g

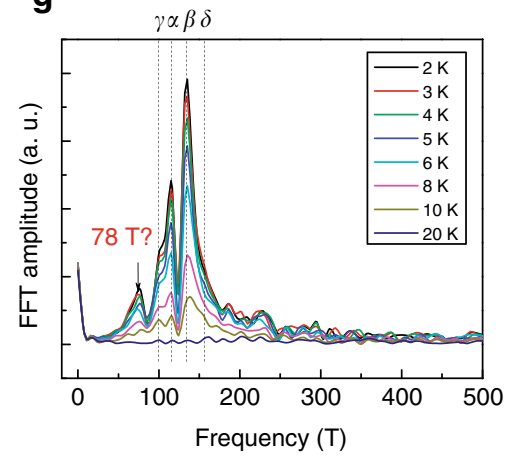

Fig. 1 Fermi-arc-induced Weyl orbit oscillations. a The calculated Weyl points and a possible Fermi arc in the $k_{z}=0$ plane of WTe ${ }_{2}^{6,13}$. The Fermi arcs are mainly along $Y$ direction. $\mathbf{b}$ Schematic of a Fermi-arc-induced Weyl orbit in a thin WTe $\mathrm{e}_{2}$ nanoribbon, in which the magnetic field is along the $z$-axis (or $c^{-}$) axis. This schematic is similar to that proposed by Potter et al. ${ }^{19}$. This Weyl orbit is formed by connecting two bulk paths along the $z$-direction through the zeroth chiral bulk Landau level (LL) and two Fermi arcs in the momentum space, on both the top and bottom surfaces. The trajectory of the Weyl orbit in real space is in the $x z$ plane, and the Weyl orbit is plotted in a combination of real space and momentum space. The Weyl points with opposite chirality are labeled as + (blue) and - (purple). c Conventional quantum oscillation orbit. $\mathbf{d}$ MR of the $b$-axis ribbon (19.4 nm thick) at different temperatures with $\mathbf{B} / / \mathrm{c}$. Inset shows a metal-insulator transition under a magnetic field of $14 \mathrm{~T}$. e The pronounced $\mathrm{SdH}$ oscillations are observed at different temperatures in the plots of $d^{2} R / d B^{2}$ vs. $B$. $\mathbf{f}$ Comparison of FFT of the data of $d^{2} R / d B^{2}$ vs. $B$ obtained from bulk WTe 2 and $b$-axis ribbon $(T=2 \mathrm{~K})$. The FFT data obtained from $\mathrm{d}^{2} R / \mathrm{d} B^{2}$ vs. $B$ for $B>8.0 \mathrm{~T}$ shows an extra frequency of $78 \mathrm{~T}$. $g$ The FFT spectra of $b$-axis ribbon at different temperatures 
type-II and has the chiral anomaly ${ }^{6,22}$. This phenomenon should be a direct evidence for type-II Weyl semimetals. Moreover, a new cyclotron motion in Weyl and Dirac semimetals, the Weyl orbit, is proposed to be formed by connecting these surface Fermi arcs via the Weyl points in the bulk crystal ${ }^{19}$. This Weyl orbit will lead to an additional frequency in the magnetic quantum oscillation spectra for experiments that probe the Fermi surfaces, such as magneto-transport measurements. The observation of the extra Weyl orbit frequency is indirect evidence, because it cannot provide the information about the type of a Weyl semimetal. It is important to note that the Weyl orbit in type-II Weyl semimetals will be slightly modified due to the absence of chiral Landau levels ${ }^{24-26}$, when the magnetic field is normal to the tilt of Weyl cone. However, the main conclusions keep the same as in type-I Weyl semimetals (Supplementary Note 1). Therefore, we are motivated to explore these two exotic properties, the evidence for type-II Weyl semimetal $\mathrm{WTe}_{2}$, through magneto-transport measurements.

In this work, we use electrical transport measurements to demonstrate that $\mathrm{WTe}_{2}$ is a type-II Weyl semimetal with topological Fermi arcs. Based on the theoretical prediction and previous experimental results, a material can be viewed as a type-II Weyl semimetal if we observe extra-quantum oscillation due to the Weyl orbit formed by a connection between Fermi arcs and bulk Weyl points ${ }^{12,19}$, and an anisotropic negative MR caused by a chiral anomaly $6,22,24-26$. Below, we show that these two characteristics of a type-II Weyl semimetal are clearly observed simultaneously on the same piece of $\mathrm{WTe}_{2}$ nanoribbons.

\section{Results}

Extra-quantum oscillation from Weyl orbit. According to the theoretical prediction, topological surface states exist only on the (001) planes of $\mathrm{WTe}_{2}{ }^{6}$. To study the transport anisotropy, we patterned nanoribbons from the longest $a$-axis and $b$-axis sides of the same slab of exfoliated $\mathrm{WTe}_{2}$ ( $a$-axis and $b$-axis ribbon, Supplementary Fig. 1). To avoid complexity caused by the extrinsic effect, such as severe scattering of the ribbon boundaries, all of the ribbons were fabricated with a width larger than $0.6 \mu \mathrm{m}$ (Supplementary Fig. 2). Moreover, as the thickness of the $\mathrm{WTe}_{2}$ ribbons decreased to $5.6 \mathrm{~nm}$, the electrical transport behavior transformed gradually from metallic to insulating; particularly, the size of MR was largely suppressed (Supplementary Fig. 3), which is consistent with previous work ${ }^{27}$. Therefore, a $b$-axis ribbon with a moderate thickness $t=19.4 \mathrm{~nm}$ ( $\sim 14$ atomic layers) was chosen for a detailed investigation of its magneto-transport properties (Supplementary Fig. 4a). The observations of a significantly large MR (>400\%) for both $\mathbf{I} / / b$ and $\mathbf{B} / / c$ at low temperatures, and of a metal-insulator transition under the high magnetic field $B=14 \mathrm{~T}$ (Fig. 1d and its inset), indicate clearly that the $19.4 \mathrm{~nm}$ thick ribbon still remains in the bulk state of $\mathrm{WTe}_{2}{ }^{18}$. Below $20 \mathrm{~K}$ (Fig. 1e), we also observe pronounced Shubnikov-de Hass ( $\mathrm{SdH})$ oscillations from the second-order derivatives, $\mathrm{d}^{2} R / \mathrm{d} B^{2}$, of the data in Fig. 1d, indicating the high quality of the nanoribbons.

To understand the data obtained from the $b$-axis nanoribbon (Fig. 1e), we carried out the same measurements on bulk single crystals with the same configuration $(\mathbf{I} / / b$ and $\mathbf{B} / / c)$. The fast Fourier transformation (FFT) of the second-order derivative of the MR obtained from both the nanoribbon and the bulk sample are compared in Fig. 1f. Clearly, the frequencies of the bulk crystal agree well with previous reports that the quantum oscillations originate from four ellipsoidal Fermi surfaces, two electron pockets $\left(\alpha\right.$ and $\beta$ ) and two hole pockets $(\gamma \text { and } \delta)^{28,29}$. Compared to the frequencies of the bulk crystal, the $b$-axis ribbon data show three interesting features: decreasing/vanishing of the peaks of the two hole pockets ( $\gamma$ and $\delta$ ); the positions of the two electron pocket peaks ( $\alpha$ and $\beta$ ) shift much more than the peaks of the two hole pockets $(\gamma$ and $\delta$ ); and the appearance of an extra peak at 78.0 T. The diminishing of the peaks at the two hole pockets indicate that the electronic transport of the ribbon is dominated by electrons, which may suggest a rise in the Fermi level of the ribbon compared to its bulk counterpart. Notably, the predicted Weyl points are about $50-80 \mathrm{meV}$ above the Fermi level ${ }^{6}$; therefore, a higher Fermi level in the nanoribbon may make it possible for us to detect the potential Weyl points through the transport measurements. Compared to the FFT data of bulk $\mathrm{WTe}_{2}$, frequencies in nanoribbons correspond to different Fermi surface sizes, i.e., shift in the chemical potential. The shift of peak positions is probably caused by the Fermi triaxial ellipsoids shrinking in volume as the ribbon thickness decreases and Fermi level increases ${ }^{30}$, which is also supported by the gradual opening of the bandgap as the thickness of $\mathrm{WTe}_{2}$ decreases (Supplementary Fig. 3). To explore the origin of the peak at 78.0 T, we analyze the data more carefully, finding that this extra peak came from the data collected under a magnetic field higher than $8.0 \mathrm{~T}$, and that only four peaks corresponding to bulk Fermi surfaces can be observed below 8.0 T. Figure $1 \mathrm{~g}$ shows the FFT data from Fig. 1d at different temperatures. The strength of the peaks decreases quickly as the temperature increases. This temperature dependence can be well-fitted by the Lifshitz-Kosevich equation, which leads to effective masses of $0.412 m_{0} \pm 0.021 m_{0}(\alpha), \quad 0.482 m_{0} \pm 0.024 m_{0}(\beta)$, and $0.253 m_{0} \pm$ $0.013 m_{0}(78.0 \mathrm{~T}$ ) (Supplementary Fig. $4 \mathrm{~b}-\mathrm{d}$ ). The effective masses of $\alpha$ and $\beta$ in the ribbon agree well with those in bulk $\mathrm{WTe}_{2}{ }^{28}$.

To understand the physics of the extra peak at 78.0 T, let us first examine whether the semi-classical Weyl orbit model ${ }^{19}$ can be applied here. In this model, a cyclotron motion formed by the chiral Landau levels and the Fermi arcs may result in an extraquantum oscillation in Dirac or Weyl semimetals; this was successfully applied to interpret the experimental observation, particularly to demonstrate the existence of the non-trivial surface states on a slab of the Dirac semimetal, $\mathrm{Cd}_{3} \mathrm{As}_{2}{ }^{12}$. Nevertheless, several calculations suggest that chiral Landau levels in type-II Weyl semimetals will be absent when the applied magnetic field $(\mathbf{B} / / z)$ is normal to the tilting direction of Weyl cone ( $Y$ direction $^{24-26}$. Therefore, the semi-classical Weyl orbit in typeII Weyl semimetals should be slightly modified. To form a Weyl orbit, the bulk Landau levels will play the role of the chiral Landau levels to connect the Fermi arcs as in the type-I and Dirac semimetals. This modification in the model of Weyl orbit will not change the main conclusions (see Supplementary Note 1 in details). If the electric field is applied along the $k_{y}(b)$ direction and the magnetic field is applied along the $z$ (or $c$ ) direction in the $b$-axis ribbon of $\mathrm{WTe}_{2}$, a complete Weyl orbit will form (Fig. 1b) and correspond to a trajectory in the $x z$ plane in real space. A unique feature of the Weyl orbit is that the magnetic field is parallel to the orbit in real space, rather than normal to the orbits of the conventional quantum oscillations (Fig. 1c). According to this model, the frequency of the quantum oscillation due to this Weyl orbit can be estimated by ref. ${ }^{19}$

$$
F_{\mathrm{S}}=\frac{E_{\mathrm{F}} k_{0}}{e \pi \bar{v}_{\mathrm{F}}}
$$

where $E_{\mathrm{F}}=\hbar \bar{v}_{\mathrm{F}} \bar{k}_{c}$ and $\bar{v}_{\mathrm{F}}=\hbar \bar{k}_{c} / \bar{m}^{*}$ are the bulk Fermi energy and Fermi velocity, respectively, of electrons sliding along the $c$ direction via the chiral bulk LLs; $k_{0}$ is Fermi-arc separation.

Therefore, to obtain the frequency $F_{\mathrm{S}}$ of the Weyl orbit in a $b$ axis ribbon, we extract $E_{\mathrm{F}}, v_{\mathrm{F}}$, and the Fermi radius $k_{c}$ from the $\mathrm{SdH}$ oscillations obtained from the bulk $\mathrm{WTe}_{2}$. The Fermi radius can be calculated using the Onsager equation $F=(\hbar / 2 \pi \mathrm{e}) S_{\mathrm{F}}{ }^{31}$, 
a

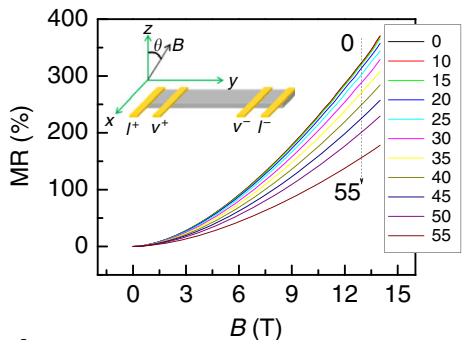

d

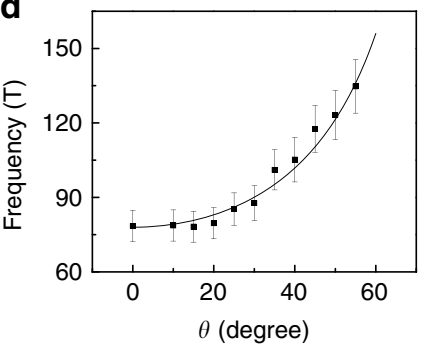

b

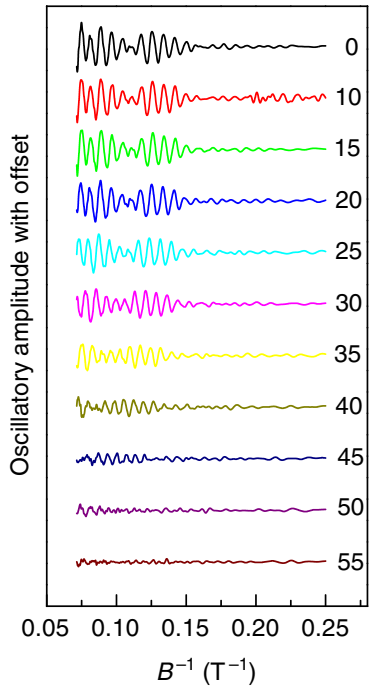

c

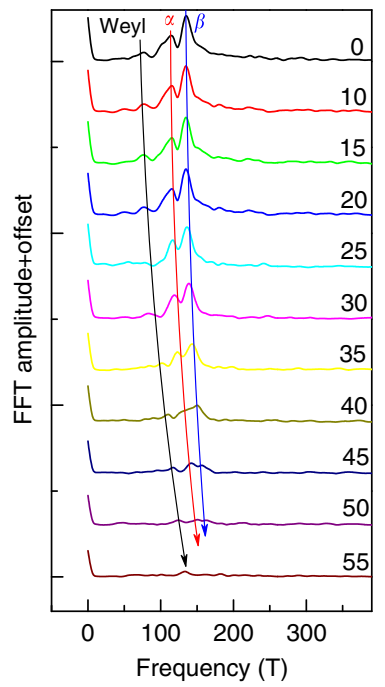

Fig. 2 Angle-dependent quantum oscillations. a The MR data from $b$-axis ribbon obtained at different angles and $T=2 \mathrm{~K}$. $\mathbf{b}$ The $\mathrm{d}^{2} R / \mathrm{d} B^{2}$ data obtained from $\mathbf{a}$ clearly shows quantum oscillations. c The evolution of quantum frequency in FFT spectra obtained from $\mathbf{b}$ as the angle increases. The arrow indicates the

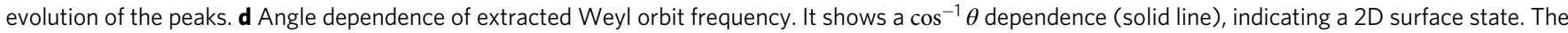
error bar of $20 \%$, including standard instrument error and FFT frequency spacing estimate, was added into the angular dependence of frequency

where $S_{\mathrm{F}}$ is the extreme cross-sectional area of the 3D Fermi surface. All four of the Fermi surface pockets mapped by quantum oscillations are triaxial ellipsoidal rather than spherical (Supplementary Fig. 5 and Supplementary Table 1). Since the electronic transport in the $\mathrm{WTe}_{2}$ ribbons is dominated by electrons, we only need the values of $k_{\mathrm{F}}$ for the two electron pockets along the $c$ direction ( $\alpha$ and $\beta$ in Fig. 1f). The radii of the two electron pockets are obtained as $k_{\alpha c}=1.19 \mathrm{~nm}^{-1}$ and $k_{\beta c}=$ $1.22 \mathrm{~nm}^{-1}$ (Supplementary Table 1). These values are in good agreement with previous quantum analyses from Seebeck measurements ${ }^{28}$. The Fermi velocity of bulk electrons in a Weyl orbit and the bulk Fermi energy are obtained as

$$
\begin{gathered}
\bar{v}_{\mathrm{F}}=\frac{\hbar \bar{k}_{c}}{\bar{m}^{*}} \approx 3.09 \times 10^{5} \mathrm{~ms}^{-1}, \\
E_{\mathrm{F}}=\hbar \bar{v}_{\mathrm{F}} \bar{k}_{c} \approx 244.5 \mathrm{meV} .
\end{gathered}
$$

Thus, we calculate the frequency of the quantum oscillation caused by this Weyl orbit using Eq. (1) as $F_{\mathrm{S}} \approx 80.6 \mathrm{~T} \pm 4.0 \mathrm{~T}$, where the value of the Fermi-arc separation $k_{0}$ is $0.032 \mathrm{~A}^{-1} \pm$ $0.002 \mathrm{~A}^{-113}$. This value agrees well with the experimental observation of $78.0 \mathrm{~T} \pm 3.9 \mathrm{~T}$. And two more sets of data with Weyl orbit frequency and effective mass analysis are shown in Supplementary Figs. 6 and 7. The observation of the coherent quantum oscillation associated with the Fermi arc requires that $\omega_{\mathrm{c}} \tau=e v_{\mathrm{F}} B \tau / 2 k_{0}>1^{19}$, i.e., the electrons must finish a complete Weyl orbit (Fig. 1b) before being scattered off. For a quantum scattering time $\bar{\tau}=(1.6 \pm 0.1) \times 10^{-13}$ s (Supplementary Fig. 8), the quantum oscillations associated with Fermi arcs can be observed only when the applied magnetic field $B>8.6 \mathrm{~T}$, which is consistent with the observation of a $78.0 \mathrm{~T}$ peak only when $B>8.0 \mathrm{~T}$ (Fig. 1f, e). Thus, we conclude that we have indeed demonstrated the existence of a Fermi-arc-induced quantum oscillation in $\mathrm{WTe}_{2}$.

Two-dimensional feature of Weyl orbit. As shown in Fig. 1b, the Weyl orbit can only be detected when a non-zero, perpendicular component of magnetic field is applied to the $a b$ plane; therefore, some two-dimensional (2D) characteristics of the Weyl orbit should be observed. Fig. 2a shows that MR decreases significantly as the angle increases from $\theta=0^{\circ}\left(\mathbf{B} / / z\right.$-axis) to $\theta=90^{\circ}(\mathbf{B} / / a b$ plane). The $\mathrm{SdH}$ oscillations at different angles are clearly observed in the plots of $\mathrm{d}^{2} R / \mathrm{d} B^{2}$ vs. $B^{-1}$ (Fig. 2b). It is evident that the $\mathrm{SdH}$ oscillations evolve with an increasing $\theta$. The frequencies of $\alpha$ and $\beta$ increase with $\theta$, as expected given the ellipsoid-like electron pockets in the bulk $\mathrm{WTe}_{2}$ (Supplementary Fig. 5). The most interesting feature in Fig. $2 \mathrm{c}$ is that the oscillation frequency of the Weyl orbit shifts faster than that of the two electron pockets, indicating that the Weyl orbit has different origin from that of the two electron pockets. The extracted extra frequency

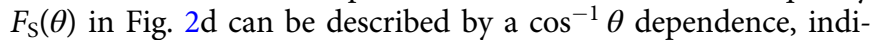
cating that the Weyl orbit is $2 \mathrm{D}$ in nature. However, to observe the frequency of a Weyl orbit at higher angles $\left(\varphi>60^{\circ}\right)$, the minimum magnetic field must be stronger than $17 \mathrm{~T}(\sim 8.6 \mathrm{~T} / \mathrm{cos}$ 60 ), which exceeds the maximum magnetic field of $14 \mathrm{~T}$ in our experiment.

Anisotropic chiral anomaly. Another crucial piece of evidence confirming the existence of type-II Weyl semimetals is the anisotropic negative longitudinal MR induced by a chiral anomaly. The negative longitudinal MR appears only when a magnetic field $(B)$ are parallel to the tilt direction of Weyl cones, but chiral Landau levels, i.e., negative longitudinal $M R$, are absent when a magnetic field is normal to the tilt direction of Weyl cones $^{6,23-26,32}$. The MR curves measured at $2 \mathrm{~K}$ with $\mathbf{B} / / a$ and $\mathbf{B} / / b$ are shown in Fig. 3a. The MR data for $\mathbf{B} / / c$ (shown in Fig. 1d) is replotted here for comparison. Although the magnetic fields are perpendicular to the current in both cases, $\mathbf{B} / / a$ and $\mathbf{B} / / c$, the positive MR ratio when $\mathbf{B} / / a$ is almost two orders of magnitude smaller than that when $\mathbf{B} / / c$, which is consistent with a previous observation in bulk $\mathrm{WTe}_{2}{ }^{33}$. This strongly anisotropic MR behavior is ascribed to the strong anisotropy in the carrier mobility ${ }^{28,33}$. As expected, we observe a negative, longitudinal MR under high magnetic fields $(B>4 \mathrm{~T})$ in the case of $\mathbf{B} / / \mathbf{I} / / b$, which strongly supports the prediction of an Adler-Bell-Jackiw anomaly in the presence of a strong magnetic field, and is in good agreement with recent works $22,23,34-36$. We carefully examined the origin of the negative MR and finally ruled out other possible mechanisms of negative MR, such as current jetting and Knudsen 


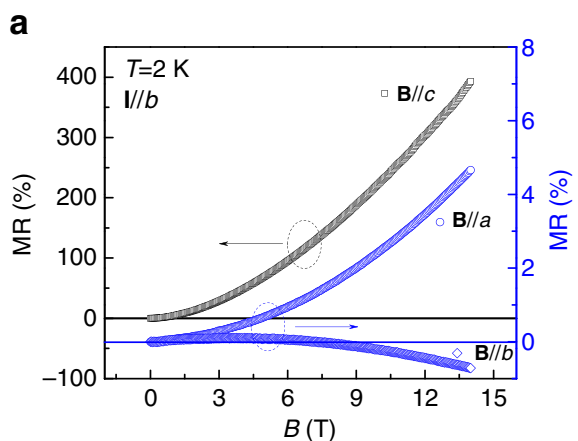

d

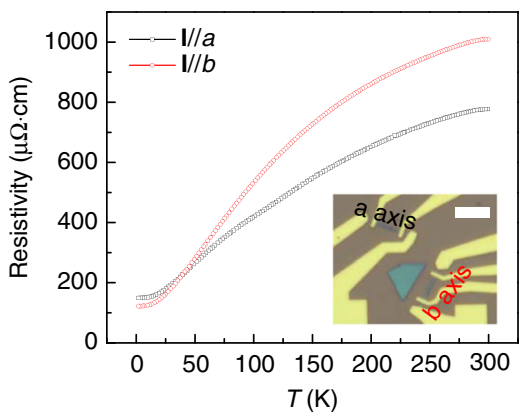

b

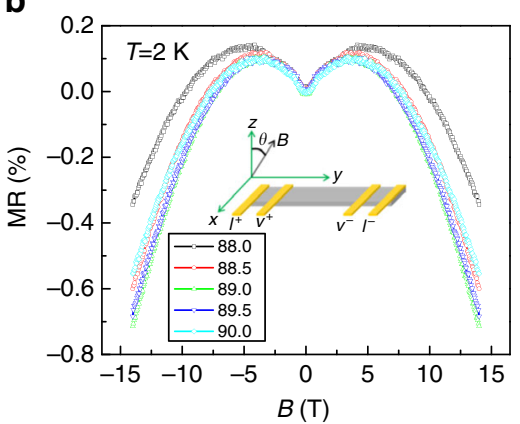

e

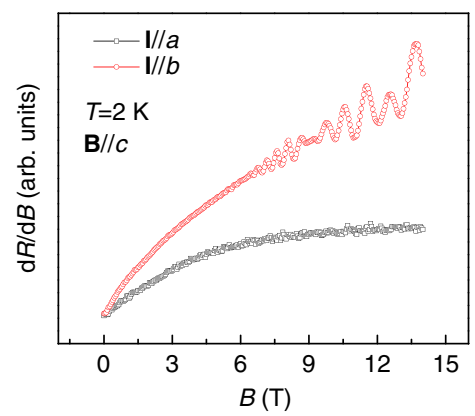

c

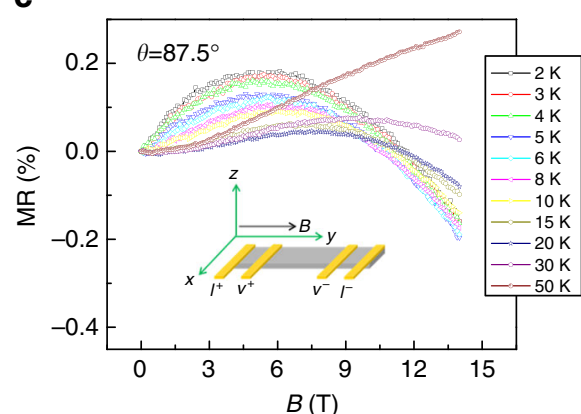

f

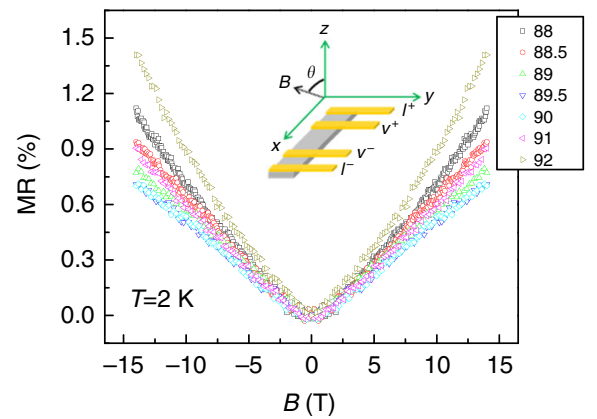

Fig. 3 Chiral-anomaly induced negative longitudinal MR. a MR data obtained along different field directions at $2 \mathrm{~K}$ show strong anisotropy in the transport properties. In all of the measurements, $\mathbf{I} / / \mathbf{b}$ in the $19.4 \mathrm{~nm}$ thick $b$-axis ribbon. $\mathbf{b}$ The field-dependent, negative and longitudinal MR induced by a chiral anomaly in $b$-axis ribbon. We note that the occurrence of the maximum negative MR at $89^{\circ}$ rather that at $90^{\circ}$ could be due to a misalignment when we glued the sample to the sample holder. c The longitudinal MR measured with $\theta=87.5^{\circ}$ at different temperatures. $\mathbf{d}$ The temperature-dependent resistivity of $19.4 \mathrm{~nm}$ thick $a$-axis and $b$-axis ribbon. The scale bar in the optical image is $5 \mu \mathrm{m}$. e Comparison of quantum oscillation in both ribbons with the magnetic field normal to the ribbon. $\mathbf{f}$ The absence of chiral-anomaly-induced negative MR in $a$-axis ribbon

effect $^{37-40}$ (see details in Supplementary Note 2 and Supplementary Figs. 9 and 10). To further confirm that the negative MR for $\mathbf{B} / / \mathbf{I} / b$ was indeed induced by a chiral anomaly, we measured the longitudinal $\mathrm{MR}$ as we slightly varied the applied field direction. As shown in Fig. $3 \mathrm{~b}$, the absolute value of the negative MR decreases quickly as the magnetic field deviates slightly from the $E$-field direction, a unique feature of a negative MR induced by a chiral anomaly ${ }^{4}$. The temperature-dependent MR measured at $\theta=87.5^{\circ}$ (Fig. 3c) indicates clearly that the chiral anomaly can survive up to $30 \mathrm{~K}$. The behavior of the magnetic field-dependent MR can be well described by the semi-classical model, in which both the chiral anomaly and the weak antilocalization (Supplementary Fig. 11) are considered ${ }^{4}$. To summarize, we have observed two unique properties of a type-II Weyl semimetal in the same piece of $b$-axis $\mathrm{WTe}_{2}$ ribbon: a quantum oscillation induced by a Fermi arc and a negative MR induced by a chiral anomaly. Therefore, we conclude that $\mathrm{WTe}_{2}$ is, indeed, a type-II Weyl semimetal.

One may argue that the observed quantum oscillation comes from the trivial surface states instead of a Weyl orbit (Fermi arcs). If the above observations really originated from the trivial surface states, we would be able to observe a similar quantum oscillation and negative MR induced by a chiral anomaly in an $a$-axis ribbon. To address this argument, we measured the magneto-transport properties on an $a$-axis ribbon fabricated from the same $\mathrm{WTe}_{2}$ slab used for the $b$-axis ribbon (Supplementary Fig. 4a). The temperature-dependent resistivity curves of the $\mathrm{WTe}_{2}$ nanoribbons are shown in Fig. 3d. A higher residual resistivity along the $a$-axis than along the $b$-axis indicates that the average carrier mobility is smaller along the $a$-axis than along the $b$-axis $\left(\rho^{-1}=\right.$ $(n+p) e \mu)$, which is consistent with the transport anisotropy observed previously in bulk WTe ${ }_{2}{ }^{28,33}$. Although we observed a large, positive $\mathrm{MR}$ in the $a$-axis ribbon at low temperatures, similar to the $b$-axis sample (Supplementary Fig. 12), we were not able to observe any trace of quantum oscillation, even after removing the linear background (Fig. 3e). The complete disappearance of all quantum oscillations, including those arising from the intrinsic electron pockets of bulk sample, may be attributed to the strong mobility anisotropy $\mu_{a}<\mu_{b}$, which is supported by the observation in Fig. $3 \mathrm{~d}^{28}$. The disappearance of the quantum oscillation caused by a Weyl orbit in the $a$-axis ribbon can be ascribed to the fact that no Weyl orbit can be formed without the presence of Fermi arcs along the $X$ direction in momentum space (Fig. 1a, b).

To further demonstrate that the Weyl points and Fermi arcs are found along the $Y$ direction in $\mathrm{WTe}_{2}$, we measured the longitudinal MR of the $a$-axis ribbon at $2 \mathrm{~K}$. As expected, no chiral-anomaly-induced negative MR was observed because the chiral Landau levels are absent ${ }^{24-26}$ when the magnetic field (B// $a)$ is normal to the tilting direction of Weyl cone $(B \perp b$ Fig. 3f). A similarly anisotropic behavior of the chiral anomaly was observed previously in $\mathrm{WTe}_{2}$ slab samples ${ }^{34}$ and was attributed to the fact that the kinetic energy is smaller than the potential energy, along the $a$-axis ( $X$ direction in momentum space) $)^{6,22,34}$. Therefore, the absence of both a Weyl orbit quantum oscillation and a chiral anomaly in $a$-axis ribbon further verifies the prediction that nontrivial topological Fermi arcs are only found along the $Y$ direction in the (001) plane of $\mathrm{WTe}_{2}$.

Thickness dependence of Weyl orbit. As theoretically predicted $^{19}$ and experimentally demonstrated ${ }^{12}$ previously, a key characteristic of the quantum oscillation from a Weyl orbit is that its amplitude decays very fast with increasing the ribbon thickness. Plotted in Fig. $4 \mathrm{a}$ are the MR data measured at $2 \mathrm{~K}$ from the $b$-axis ribbons with thickness varying from 12.6 to $40.6 \mathrm{~nm}$. The 
a
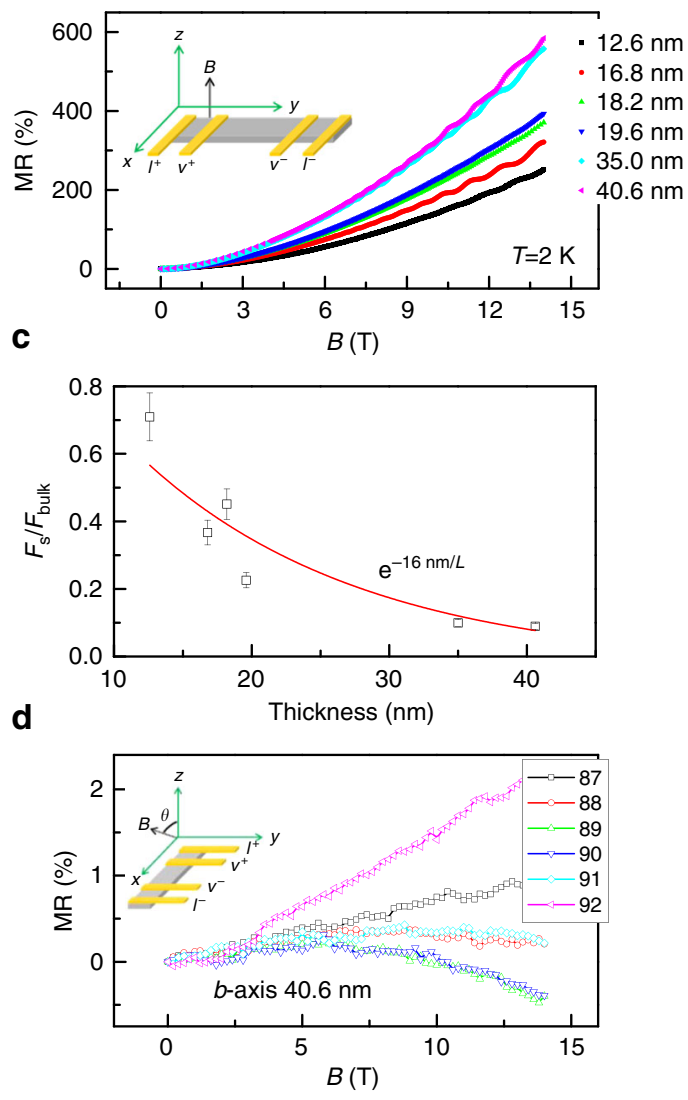

b

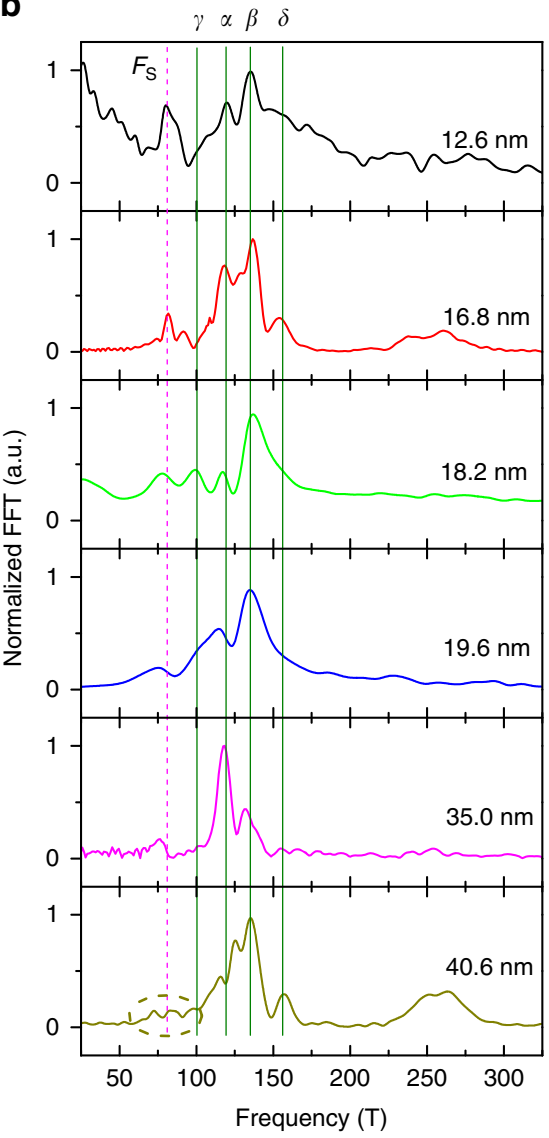

Fig. 4 Thickness-dependent Weyl orbit oscillations. a The MR of $b$-axis ribbon with different thicknesses. $\mathbf{b}$ The FFT spectra of $b$-axis ribbons with different thicknesses, in which the spectra are normalized with respect to the strongest frequency of electron pocket $\beta$. The Weyl orbit frequency almost vanishes in $40.6 \mathrm{~nm}$ thick ribbon. c Thickness dependence of the relative amplitude of the Weyl orbit oscillation and the bulk oscillation with respect to peak $\beta$. The thickness dependence of relative amplitude can be fitted by $e^{-L_{0} / L}$, with $L_{0}=16.0 \mathrm{~nm}$. The fitted value of $L_{0}$ is in the same magnitude of the mean free path (c-axis) $\sim 48.0 \mathrm{~nm}$. The $20 \%$ error bar was added given to the FFT frequency spacing estimate. $\mathbf{d}$ The chiral-anomaly-induced negative MR in $40.6 \mathrm{~nm}$ thick $b$-axis ribbon

MR increases greatly as the thickness of the sample increases. This enhanced MR in thicker ribbons can be ascribed to the increased mobility ${ }^{27}$ and the decreased Fermi level. Both factors lead to a larger $n-p$ compensated $\mathrm{MR}^{18,27}$. To clearly demonstrate how the quantum oscillation of the Weyl orbit depends on the thickness of the ribbon, we normalized the FFT spectra with respect to the strongest bulk Fermi surface frequency (Fig. 4b). It is evident in Fig. $4 \mathrm{~b}$ that the Weyl orbit frequency $(\sim 78 \mathrm{~T})$ is very strong in the $12.6 \mathrm{~nm}$ sample, and it decays quickly as the thickness of the ribbon increases; nearly vanishing when the thickness is increased to $40.6 \mathrm{~nm}$ (dashed circle in Fig. 4b). This fast decay can be seen more clearly in Fig. 4c, in which the ratio of $F_{\mathrm{S}} / F_{\max }$ is plotted vs. thickness. Although the extra-quantum oscillation could hardly be observed in the thickest nanoribbon $(40.6 \mathrm{~nm})$, the negative MR induced by a chiral anomaly in $\mathbf{B} / \mathbf{I} / / b$ was large enough (about $0.5 \%$ at $14 \mathrm{~T}$ ) to be detected (Fig. $4 \mathrm{~d}$ ). Even though it was smaller in the $40.6 \mathrm{~nm}$ thick nanoribbon than that in the $19.4 \mathrm{~nm}$ thick ribbon, the negative MR strongly indicates that the Weyl points along $\mathbf{Y}$ direction still play an important role in the chiral anomaly transport. To further support our conclusion that type-II Weyl points and a Fermi arc exist in $\mathrm{WTe}_{2}$, we analyzed the vanishing Weyl orbit quantum oscillation in more detail in the $40.6 \mathrm{~nm}$ thick sample. Given the Fermi velocity $\bar{v}_{\mathrm{F}}=3.09 \times 10^{5} \mathrm{~ms}^{-1}$, the time required for an electron to travel between the top and bottom surfaces along the $c$-axis can be approximated as $t \approx L / v_{\mathrm{F}}=40.6 \mathrm{~nm} / 3.09 \times 10^{5} \mathrm{~ms}^{-1}$ $\approx 1.3 \times 10^{-13} \mathrm{~s}$, which is quite close to the coherent quantum scattering time of both electron pockets, $\bar{\tau}=(1.6 \pm 0.1) \times 10^{-13} \mathrm{~s}$ (Supplementary Fig. 8). Not surprisingly, the Fermi-arc-induced quantum oscillation at $\sim 78 \mathrm{~T}$ vanishes (Fig. $4 \mathrm{~b}$ ) in the $40.6 \mathrm{~nm}$ thick $b$-axis ribbon. The fast decay of the Weyl orbit agrees well with both the theoretical predictions and the experimental results obtained from slabs of $\mathrm{Cd}_{3} \mathrm{As}_{2}{ }^{12,19}$. The thickness-dependent decay of amplitude of Weyl orbit could be reasonably well-fitted by exponential behavior $\left(e^{-L_{0} / L}\right)$ with $L_{0} \approx 16.0 \mathrm{~nm}$, which is in the same magnitude of the mean free path $l \approx 48.0 \mathrm{~nm}(c$-axis, $\left.v_{\mathrm{F}} \bar{\tau} \approx 48.0 \mathrm{~nm}\right)$.

\section{Discussion}

Therefore, to observe the topologically protected Fermi-arcinduced quantum oscillation, the sample thickness must be large enough to ensure both metallic behavior $(>10 \mathrm{~nm})$ and $\mathrm{SdH}$ oscillation, and the time spent crossing the sample ribbon must not exceed the coherent quantum scattering time (Supplementary Figs. 13 and 14 and Supplementary Note 1). Although we can observe the decay of oscillation amplitude of Weyl orbit quantum oscillations on thickness, it is still difficult to observe the effect of thickness on Weyl orbit quantum oscillation phase ${ }^{12}$ (see Supplementary Note 3 and Supplementary Fig. 15). Finally, we have also examined if this additional quantum oscillation is a consequence of Klein tunneling, since it was predicted theoretically 
that electrons may tunnel through a single type-II Weyl point with the mechanism of Klein tunneling ${ }^{41}$. Unfortunately, we were unable to exclude or demonstrate the existence of this quantum oscillation (Supplementary Note 4 and Supplementary Fig. 16).

To conclude, although $\mathrm{WTe}_{2}$ was suggested to be a type-II topological Weyl semimetal, a full experimental confirmation of this theory has been lacking, owing to the limited resolution of ARPES. We have presented strong experimental evidence to confirm that $\mathrm{WTe}_{2}$ is indeed a type-II topological Weyl semimetal by observing two unique properties: an extra-quantum oscillation arising from a Weyl orbit and an anisotropic negative MR resulted from a chiral anomaly. Our work and the work on the Dirac semimetal $\mathrm{Cd}_{3} \mathrm{As}_{2}$, together with the theoretical prediction, have demonstrated that the magneto-transport measurements, including both the direct evidence, the negative longitudinal magnetoresistance induced by anisotropic chiral anomaly and the indirect evidence, Weyl orbit, are reliable and convenient to explore the existence of type-II Weyl semimetals.

\section{Methods}

Device fabrication. A schematic of the device fabrication process is shown in Supplementary Fig. 1. First, the multilayered WTe $\mathrm{W}_{2}$ slabs were exfoliated from the CVD-grown single crystal, which was bought from Hqgraphene Company. The inplane crystal orientation ( $a$-axis and $b$-axis) was determined using the angledependent polarized microscopic Raman spectrum (Hariba LABRAM HR spectrometer), as shown in Supplementary Fig. 1b. An excitation laser with a wavelength of $473 \mathrm{~nm}$ was used. To avoid oxidation caused by the laser, a filter was inserted to make sure that the laser power was below $300 \mu \mathrm{W}$ during the Raman measurement. To minimize oxidation from the surrounding air, the newly exfoliated $\mathrm{WTe}_{2}$ slabs on a $\mathrm{SiO}_{2}(285 \mathrm{~nm}) / \mathrm{Si}$ substrate were placed in a vacuum chamber immediately after the Raman measurement.

To measure the transport properties with current flowing along the $a$ and $b$ axes, $\mathrm{WTe}_{2}$ nanoribbons with widths of $0.6-1 \mu \mathrm{m}$ and lengths of $10-15 \mu \mathrm{m}$ were shaped using the standard E-beam lithography (EBL) process, which was followed by a reactive etching with $\mathrm{Cl}_{2}$ gas to remove the excess $\mathrm{WTe}_{2}$ slabs, as shown in Supplementary Fig. 1c. Finally, small electrodes $\mathrm{Ti}(10 \mathrm{~nm}) / \mathrm{Au}(70 \mathrm{~nm})$ were shaped using the EBL process again, which was followed by E-beam evaporation. Supplementary Fig. 1 includes a schematic of the nanofabrication method. Another slab ( 700 nm, Supplementary Fig. 11) was fabricated using a focused ion beam attached to the scanning electron microscope (SEM, Helios 650, FEI).

Transport measurement. The thickness of the $\mathrm{WTe}_{2}$ slabs and the ribbon-like slab were determined using an atomic force microscope (AFM). The morphology of the devices was characterized by scanning electron microscopy (SEM Nova Nano 630, FEI). The magneto-transport of the ribbon-like devices with different crystal configurations was measured in the physical properties measurement sys tem (Dynacool) by quantum design, with a temperature range of $2-300 \mathrm{~K}$ and a magnetic field up to $14 \mathrm{~T}$, during which a sample rotator helped to change the direction of the magnetic field with respect to the current. The resistance was measured using the ac lock-in method with the standard four-terminals. An AC current of $1 \mu \mathrm{A}$ was applied to avoid overheating and damaging the nanoribbons. The magnetoresistance ratio of our samples was defined as $\mathrm{MR}=\left(R_{B}-R_{0}\right) / R_{0}$. To analyze the quantum oscillation, we used $\mathrm{d} R / \mathrm{d} B$ or $\mathrm{d}^{2} R / \mathrm{d} B^{2}$ to remove the large linear magnetoresistance background, which was caused by the nearly compensated $n-p$ compensation in semimetallic $\mathrm{WTe}_{2}{ }^{42}$.

Data availability. The data that support the findings of this study are available from the corresponding author upon request.

Received: 7 June 2017 Accepted: 14 November 2017

Published online: 15 December 2017

\section{References}

1. Fang, Z. et al. The anomalous Hall effect and magnetic monopoles in momentum space. Science 302, 92-95 (2003).

2. Gorbar, E., Miransky, V. \& Shovkovy, I. Engineering Weyl nodes in Dirac semimetals by a magnetic field. Phys. Rev. B 88, 165105 (2013).

3. Liu, Z. et al. Discovery of a three-dimensional topological Dirac semimetal, $\mathrm{Na}_{3} \mathrm{Bi}$. Science 343, 864-867 (2014).

4. Huang, $X$. et al. Observation of the chiral-anomaly-induced negative magnetoresistance in 3D Weyl semimetal TaAs. Phys. Rev. X 5, 031023 (2015).
5. Liang, T. et al. Ultrahigh mobility and giant magnetoresistance in the Dirac semimetal $\mathrm{Cd}_{3} \mathrm{As}_{2}$. Nat. Mater. 14, 280-284 (2015).

6. Soluyanov, A. A. et al. Type-II Weyl semimetals. Nature 527, 495-498 (2015).

7. Xiong, J. et al. Evidence for the chiral anomaly in the Dirac semimetal $\mathrm{Na}_{3} \mathrm{Bi}$. Science 350, 413-416 (2015).

8. Bruno, F. Y. et al. Observation of large topologically trivial Fermi arcs in the candidate type-II Weyl semimetal WTe 2 . Phys. Rev. B 94, 121112 (2016).

9. Deng, K. et al. Experimental observation of topological Fermi arcs in type-II

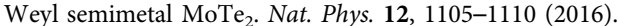

10. Feng, B. et al. Spin texture in type-II Weyl semimetal WTe 2 . Phys. Rev. B 94, 195134 (2016).

11. Huang, L. et al. Spectroscopic evidence for a type II Weyl semimetallic state in $\mathrm{MoTe}_{2}$. Nat. Mater. 15, 1155-1160 (2016).

12. Moll, P. J. et al. Transport evidence for Fermi-arc-mediated chirality transfer in the Dirac semimetal $\mathrm{Cd}_{3} \mathrm{As}_{2}$. Nature 535, 266-270 (2016).

13. Wang, C. et al. Observation of Fermi arc and its connection with bulk states in the candidate type-II Weyl semimetal WTe 2 . Phys. Rev. B 94, 241119 (2016).

14. Wu, Y. et al. Observation of Fermi arcs in the type-II Weyl semimetal candidate $\mathrm{WTe}_{2}$. Phys. Rev. B 94, 121113 (2016).

15. Jiang, J. et al. Signature of type-II Weyl semimetal phase in $\mathrm{MoTe}_{2}$. Nat. Commun. 8, 13973 (2017).

16. Li, Q. et al. Chiral magnetic effect in $\mathrm{ZrTe}_{5}$. Nat. Phys. 12, 550-554 (2016).

17. Keum, D. H. et al. Bandgap opening in few-layered monoclinic MoTe $\mathrm{Mat}_{2}$. Phys. 11, 482-486 (2015)

18. Ali, M. N. et al. Large, non-saturating magnetoresistance in $\mathrm{WTe}_{2}$. Nature $\mathbf{5 1 4}$ 205-208 (2014).

19. Potter, A. C., Kimchi, I. \& Vishwanath, A. Quantum oscillations from surface Fermi arcs in Weyl and Dirac semimetals. Nat. Commun. 5, 5161 (2014).

20. Xu, S. Y. et al. Discovery of Lorentz-violating type II Weyl fermions in LaAlGe. Sci. Adv. 3, el603266 (2017).

21. Belopolski, I. et al. Fermi arc electronic structure and Chern numbers in the type-II Weyl semimetal candidate $\mathrm{Mo}_{x} \mathrm{~W}_{1-x} \mathrm{Te}_{2}$. Phys. Rev. B 94, 085127 (2016).

22. Lv, Y.-Y. et al. Experimental observation of anisotropic Adler-Bell-Jackiw anomaly in type-II Weyl semimetal WTe ${ }_{1.98}$ crystals at the quasiclassical regime. Phys. Rev. Lett. 118, 096603 (2017).

23. Nielsen, H. B. \& Ninomiya, M. The Adler-Bell-Jackiw anomaly and Weyl fermions in a crystal. Phys. Lett. B. 130, 389-396 (1983).

24. Tchoumakov, S., Civelli, M. \& Goerbig, M. O. Magnetic-field-induced relativistic properties in type-i and type-II Weyl semimetals. Phys. Rev. Lett 117, 086402 (2016)

25. Udagawa, M. \& Bergholtz, E. J. Field-selective anomaly and chiral mode reversal in type-II Weyl materials. Phys. Rev. Lett. 117, 086401 (2016).

26. Yu, Z. M., Yao, Y. G. \& Yang, S. Y. A. Predicted unusual magnetoresponse in type-II Weyl semimetals. Phys. Rev. Lett. 117, 077202 (2016).

27. Wang, L. et al. Tuning magnetotransport in a compensated semimetal at the atomic scale. Nat. Commun. 6, 8892 (2015).

28. Zhu, Z. et al. Quantum oscillations, thermoelectric coefficients, and the fermi surface of semimetallic WTe $e_{2}$. Phys. Rev. Lett. 114, 176601 (2015).

29. Cai, P. et al. Drastic pressure effect on the extremely large magnetoresistance in $\mathrm{WTe}_{2}$ : Quantum oscillation study. Phys. Rev. Lett. 115, 057202 (2015).

30. Xiang, F.-X. et al. Thickness dependent electronic structure in $\mathrm{WTe}_{2}$ thin films Preprint at https://arxiv.org/abs/1703.02741 (2017)

31. Shoenberg, D. Magnetic Oscillations in Metals. (Cambridge University Press, Cambridge, 2009).

32. Son, D. \& Spivak, B. Chiral anomaly and classical negative magnetoresistance of Weyl metals. Phys. Rev. B 88, 104412 (2013).

33. Zhao, Y. et al. Anisotropic magnetotransport and exotic longitudinal linear magnetoresistance in $\mathrm{WTe}_{2}$ crystals. Phys. Rev. B 92, 041104 (2015).

34. Wang, Y. et al. Gate-tunable negative longitudinal magnetoresistance in the predicted type-II Weyl semimetal WTe 2 . Nat. Commun. 7, 13142 (2016).

35. Lv, B. et al. Observation of Weyl nodes in TaAs. Nat. Phys. 11, 724-727 (2015)

36. Kim, H.-J. et al. Dirac versus Weyl fermions in topological insulators: AdlerBell-Jackiw anomaly in transport phenomena. Phys. Rev. Lett. 111, 246603 (2013).

37. Arnold, F. et al. Negative magnetoresistance without well-defined chirality in the Weyl semimetal TaP. Nat. Commun. 7, 11615 (2016).

38. Dos Reis, R. et al. On the search for the chiral anomaly in Weyl semimetals: The negative longitudinal magnetoresistance. New. J. Phys. 18, 085006 (2016).

39. Thornton, T., Roukes, M., Scherer, A. \& Van de Gaag, B. P. Boundary scattering in quantum wires. Phys. Rev. Lett. 63, 2128-2131 (1989).

40. De Jong, M. \& Molenkamp, L. Hydrodynamic electron flow in high-mobility wires. Phys. Rev. B 51, 13389-13402 (1995)

41. O'Brien, T., Diez, M. \& Beenakker, C. Magnetic breakdown and Klein tunneling in a type-II Weyl semimetal. Phys. Rev. Lett. 116, 236401 (2016).

42. Du, X., Tsai, S.-W., Maslov, D. L. \& Hebard, A. F. Metal-insulator-like behavior in semimetallic bismuth and graphite. Phys. Rev. Lett. 94, 166601 (2005) 


\section{Acknowledgements}

We'd like to thank Prof. Xi Dai, institute of Physics (Beijing), for his critical reading and useful discussion in the revision of the manuscript. We thank Y. Yang for the assistance on polarized Raman, and Z. P. Hou for the useful discussion. The research reported in this publication was supported by funding from King Abdullah University of Science and Technology (KAUST). P.L. acknowledges the financial support of CRF-2015-SENSORS-2709 (KAUST).

\section{Author contributions}

P.L. conceived the idea, designed the experiments. P.L., H.N.A., and X.X.Z. wrote the manuscript. P.L. performed the sample fabrication and transport measurements. X.H. made the AFM measurement. Z.M.Y. and S.A.Y. made the calculation on Landau levels. Q.Z. and Y.W. made the transport measurement setup. C.X. performed the polarized Raman analysis on the samples. P.L., Y.W., and Z.Y.Z. performed the analysis on quantum oscillation. X.X.Z. and all authors contributed to the discussion of the results and improvement of the manuscript.

\section{Additional information}

Supplementary Information accompanies this paper at https://doi.org/10.1038/s41467017-02237-1.

Competing interests: The authors declare no competing financial interests.
Reprints and permission information is available online at http://npg.nature.com/ reprintsandpermissions/

Publisher's note: Springer Nature remains neutral with regard to jurisdictional claims in published maps and institutional affiliations.

Open Access This article is licensed under a Creative Commons Attribution 4.0 International License, which permits use, sharing, adaptation, distribution and reproduction in any medium or format, as long as you give appropriate credit to the original author(s) and the source, provide a link to the Creative Commons license, and indicate if changes were made. The images or other third party material in this article are included in the article's Creative Commons license, unless indicated otherwise in a credit line to the material. If material is not included in the article's Creative Commons license and your intended use is not permitted by statutory regulation or exceeds the permitted use, you will need to obtain permission directly from the copyright holder. To view a copy of this license, visit http://creativecommons.org/ licenses/by/4.0/.

(C) The Author(s) 2017 